\title{
Existe-t-il une signification sociale stable et univoque de la palatalisation/affrication en français ? Étude sur la perception de variantes non standard
}

\author{
Trimaille, C. \\ Lidilem, Université Stendhal, Grenoble 3 \& LIMSI \\ cyril.trimaille@u-grenoble3.fr \\ Candea, $\mathrm{M}$. \\ EA1483-CLESTHIA, Université Sorbonne nouvelle, Paris 3 \\ maria.candea@univ-paris3.fr \\ Lehka-Lemarchand, I. \\ LiDiFra, Université de Rouen et LIMSI CNRS \\ iryna.lehka-lemarchand@univ-rouen.fr
}

\section{Introduction}

Les variantes de français parlé en France hexagonale sont relativement hiérarchisées et s'organisent en fonction d'un ensemble de pratiques considéré comme «le standard » et jouissant d'un prestige très fortement ancré dans les représentations communes. Malgré cela, des observations récentes menées dans divers contextes urbains (en région parisienne - La Courneuve, Fontenay-sous-Bois, ou dans d'autres villes comme Marseille, Grenoble, Valence, Rouen) ont montré que certaines prononciations non standard sont fréquentes et peut-être même en augmentation (Jamin, Trimaille et Gasquet-Cyrus 2006, LehkaLemarchand 2007...). Parmi celles-ci, les phénomènes de palatalisation et/ou affrication de consonnes dentales $/ \mathrm{t}, \mathrm{d} /$ (mais aussi vélaires $/ \mathrm{k}, \mathrm{g} /$ ) ont été documentés par des études récentes qui leur attribuent le statut de marqueurs sociolinguistiques (Jamin 2005). C'est spécifiquement à la perception de $/ \mathrm{t} /$ et $/ \mathrm{d} /$ palatalisés devant les voyelles fermées /i, y/ que cette étude s'intéresse.

Acoustiquement, la palatalisation peut générer un continuum de réalisations allant de ce que Fónagy [1989] appelait une " mouillure » (un léger [j] après la consonne, comme dans [vwat ${ }^{j} \mathrm{yR}$ ]) jusqu'à une affrication qui aboutit à un [t] , comme dans la prononciation [vwat ${ }^{\dagger} \mathrm{y}$ ]. Nous noterons PalAff ces phénomènes de palatalisation allant jusqu'à l'affrication.

Ces variantes ont en premier lieu été observées comme caractéristiques d'habitudes de prononciation de locuteurs de «classes populaires $»^{1}$, plus particulièrement issues de l'immigration post-coloniale, mais elles ont par la suite été attestées dans certaines productions de locuteurs appartenant aux classes moyennes (Devilla \& Trimaille, 2010), voire moyennes supérieures (Trimaille 2010). A la lumière de ces résultats, la question se pose donc de savoir si ces variantes sont en phase de diffusion depuis les populations jeunes d'origine immigrée et populaire, vers les locuteurs des classes moyennes y compris n'ayant pas d'ascendance immigrée depuis plusieurs générations. Autrement dit, on peut se demander si la PalAff est un changement en cours (d'en bas) ou bien si elle demeure fortement associée aux populations d'origine immigrée, jeunes notamment, et vivant dans les ensembles urbains périphériques. En effet, deux tendances opposées semblent se manifester en parallèle.

D'un côté, la présence de PalAff dans la parole de locuteurs haut placés sur l'échelle du prestige social conforte les hypothèses formulées par une étude pilote précédente (Trimaille 2010) qui suggérait que ce phénomène n'a pas encore atteint le niveau conscient car il n'est pas perçu spontanément et consciemment par des locuteurs du tout venant, ni ouvertement stigmatisé de manière stable (exception faite de Marseille et ses environs cf. Jamin, Trimaille \& Gasquet, 2006). Nous avons par exemple remarqué qu'une légère PalAff se fait entendre dans la parole de plusieurs journalistes de chaines radiophoniques ou 
télévisuelles nationales, mais également dans les conversations modèle enregistrées pour accompagner un manuel d'apprentissage du français comme langue étrangère (Alter Ego, paru chez Hachette, 2010).

D'un autre côté, nous avons pu répertorier quelques transcriptions explicites de l'affrication utilisées pour caricaturer la prononciation de locuteurs jeunes et/ou d'origine nord-africaine, même si ces notations explicites sont pour le moment rares (sauf à Marseille). A titre d'exemple, dans une bande dessinée mettant en scène des jeunes parisiens ou banlieusards populaires, les mots interdites, tu et petites étaient graphiés interdchites, tchu et petchites (ces graphies tch/dch se trouvant seulement dans les deux dernières planches du volume, 2 reproduites dans Charlie Hebdo en 2010); ou encore, le titre d'une pièce humoristique jouée dans différentes villes de France de 2006 à 2011 contenait les mots Fatima et dire avec les graphies Fatchima et djire (Figure 1).

Figure 1:

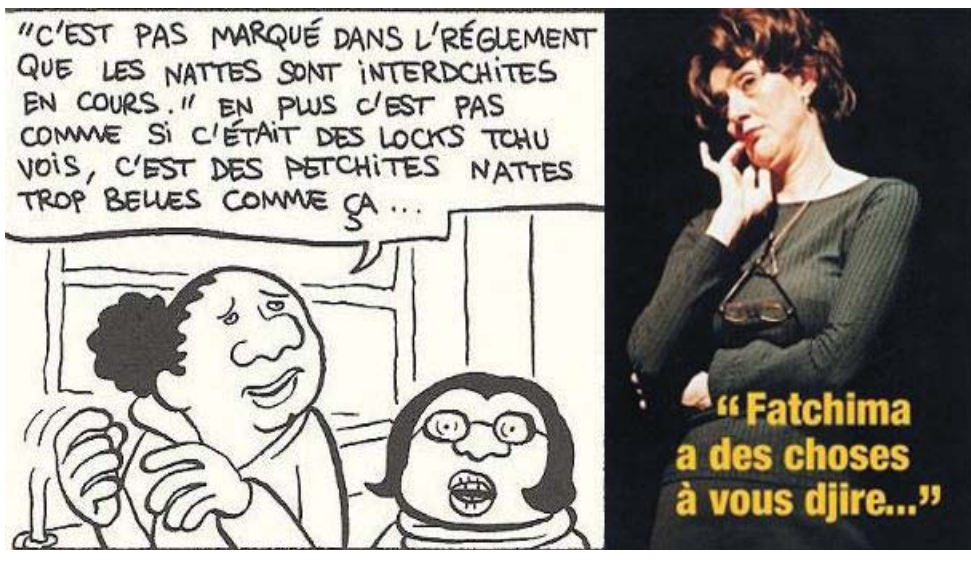

Exemples de transcriptions.

“La Vie secrète des jeunes”, R. Sattouf (gauche). Affiche d'un spectacle humoristique de S. Belhaddad (droite).

Ces remarques nous conduisent donc vers deux hypothèses opposées. Selon la première nous assistons à un début de changement phonétique, pouvant aboutir à une situation similaire à celle qui caractérise la prononciation québécoise de $/ \mathrm{t}, \mathrm{d} /$ devant voyelles fermées, favorisé par une conscience très limitée du phénomène de PalAff. Au contraire, selon la seconde hypothèse nous serions face à un processus de stéréotypisation de la PalAff ou pour le moins à une augmentation de la perception consciente des variantes affriquées, les plus saillantes, avec une sorte d'effet boule de neige sur la perception : dans ce cas, quelques locuteurs (dont certains ayant une audience large) perçoivent le phénomène et le thématisent discursivement, ce qui a pour effet d'augmenter la saillance perceptive du phénomène et d'en diffuser la conscience et la stéréotypisation.

C'est pourquoi, pour examiner la pertinence de ces deux hypothèses et les deux tendances évolutives possibles, nous souhaitons tenter de déterminer :

1) si la présence de PalAff a une influence, consciente ou non, sur l'évaluation et la catégorisation des productions des locuteurs par des auditeurs lorsqu'ils doivent évaluer l'acceptabilité de ces dernières dans des contextes formels précis

2) et, si tel était le cas, quel(s) type(s) d'attributions causales et de significations sociales la présence de PalAff dans des productions formelles susciterait-elle. 


\section{Choix méthodologiques}

En nous inspirant du travail fondateur de Labov (1972), nous avons choisi de faire écouter à des auditeurs/juges des lectures d'informations radiodiffusées. Il nous est en effet apparu que le consensus très large autour de la nécessité de réduire la présence de différentes variantes de prononciation du français dans les diffusions des radios généralistes nationales nous permettra de considérer ce cadre comme formel et de d'utiliser pour tester la conception que les auditeurs se font de la prononciation "standard » institutionnelle. Nous avons tenu compte des hypothèses formulées par Castellotti et Robillard 2003 à l'issue d'une enquête avec un protocole assez proche du nôtre ; les deux auteurs mettaient en avant une possible norme de prononciation particulièrement exigeante que construiraient les jeunes auditeurs pour décrire leurs attentes de la part des présentateurs TV. Celles-ci étaient décrites par Castellotti et Robillard comme relevant de l"inatteignable, de l'ordre de l'irréel ou fantasmé", tandis que les attentes du même public de la part d'un professeur, autre métier pourtant théoriquement réservé aux locuteurs légitimes, se révélaient être bien plus souples. Nous pensons que les attentes des auditeurs en matière de prononciation sont sensiblement équivalentes pour les présentateurs de journaux TV et ceux des journaux radiophoniques sur les chaines généralistes publiques. Par ailleurs, nous avons postulé que les mêmes auditeurs/juges ont des attentes et des exigences de «standardité $»^{2}$ moins strictes s'agissant de radios jeunes ou de radios communautaires, ce qui pourrait nous permettre de tester l'acceptabilité de variantes non standard, tout en restant dans le même contexte formel de la lecture d'informations radiodiffusées.

Pour mener en parallèle une étude plus qualitative et compréhensive qui permette d'éclairer les réponses données à des questions fermées, nous avons également construit un protocole de test par focalisation graduelle (discussion semi-guidée en focus group) afin de vérifier si le raccourcissement progressif des stimulus, visant à concentrer l'attention des auditeurs, conduit ou non à une augmentation de la perception de PalAff comme marqueur ; cette partie de notre protocole est encore en cours et ne sera pas décrite ici (cf. Lehka-Lemarchand, Candea, Trimaille, communication orale AFLS septembre 2011).

Le protocole de la partie quantitative de notre test de perception, constituée de questions fermées, vise à faire produire des jugements communs, ordinaires, sur la variation phonétique en français. Tout d'abord, nous avons conçu une dépêche radio que nous avons donnée à lire à plusieurs locuteurs, de manière à obtenir des styles de prononciation caractérisés par des niveaux différents de présence de PalAff. Ensuite, nous avons quantifié les niveaux de PalAff produits, classé les lectures en fonction de ces niveaux, et nous les avons utilisées comme stimulus, présentés dans un ordre aléatoire pour notre test.

\subsection{Stimulus}

La totalité de notre matériel utilisé pour l'expérimentation actuelle est constituée de 7 enregistrements différents de dépêches radio.

Le premier est un extrait de journal radiophonique véritablement diffusé sur Radio France Internationale (désormais RFI), qui est une chaine publique internationale d'informations ; nous l'avons utilisé comme item de contrôle car il constituait une sorte de référence pour le style de prononciation, avec un taux de PalAff quasiment nul (cf. Tableau 1, infra).

L'autre texte de dépêche rédigé par nos soins a été lu et enregistré six fois, mis en scène de manière identique (virgule musicale de début et de fin); ces lectures ont été produites par cinq locuteurs différents. Le texte de notre dépêche est composé de cinq phrases et contient 18 contextes de réalisation possible de PalAff, plus précisément 18 contextes où un $/ \mathrm{t} /$ ou un $/ \mathrm{d} /$ se retrouve immédiatement suivi par un $/ \mathrm{i}, \mathrm{j}, \mathrm{y} /$ (nous avons reproduit ce texte en annexe). La durée totale de la dépêche lue (et donc la durée des stimulus) varie entre 31,7 et 37,7 secondes, selon le rythme de chaque locuteur, ce qui représente une durée conséquente pour un test d'écoute. Un de nos objectifs étant de susciter une tâche de perception de la PalAff dans des conditions aussi naturelles que possible, nous avons souhaité éviter absolument, pour cette partie de notre protocole, d'utiliser des enregistrements trop courts, inférieurs à $10 \mathrm{~s}$, car ceux-ci activent des tâches cognitives spécifiques (Ohala \& Gilbert 1979, Morange \& Candea, 2010). Nous avons également souhaité laisser suffisamment de temps à nos auditeurs pour une bonne évaluation globale des 
extraits, tenant compte de la fréquence des variantes non standard et de leurs co-occurrences ; les extraits longs permettent des évaluations plus fines.

\subsection{Niveaux de palatalisation/affrication des stimulus}

Le classement des stimulus en fonction de leur taux de palatalisation/affrication a été établi par les auteurs et confirmé par un autre phonéticien ${ }^{3}$. Chaque expert a noté sur une échelle de 0 à 2 chaque production de $/ \mathrm{t} / \mathrm{ou} / \mathrm{d} / \mathrm{devant} / \mathrm{i}, \mathrm{j}, \mathrm{y} /$ selon les règles suivantes :

0 : prononciation standard, consonne occlusive simple

0,5 : prononciation légèrement palatalisée

1 : prononciation fortement palatalisée

$1,5:$ prononciation légèrement affriquée

2 : prononciation fortement affriquée

Pour chaque extrait, un taux moyen de PalAff a été calculé en divisant la somme des scores obtenus par le nombre de contextes possibles (ce qui pouvait donc aboutir à un taux moyen compris entre 0 et 2 ). Ces taux sont reportés dans le Tableau 1 infra selon un ordre croissant, en commençant par l'item témoin suivi des extraits de 1 à $6^{4}$. Après cet encodage, nous avons distingué trois degrés de PalAff qui permettent de regrouper les taux moyens (globaux) relativement proches .

\subsection{Locuteurs enregistrés}

Une de nos locutrices, nous l'avons dit, est une journaliste confirmée de RFI : nous avons podcasté l'enregistrement de la dépêche sur le site de la chaine. La lectrice de l'Extrait 1 (Virginie) est une professeure des écoles tandis que le lecteur de l'Extrait 3 (Hugo) est un jeune journaliste de radio. Les autres locuteurs sont étudiants ou élèves. Celle de l'Extrait 4 (Samia) est une étudiante algérienne, séjournant depuis peu de temps en France, et ayant un léger accent maghrébin. Celle des Extraits 2 et 5 (Valentine) est étudiante en fin de master de sciences du langage à Grenoble ; comme elle est capable de contrôler en partie sa prononciation des occlusives, elle a produit deux lectures différentes : une fois avec un fort taux de PalAff (Extrait 5) et une autre fois avec le taux le plus faible qu'elle pouvait réaliser (Extrait 2). Enfin la lectrice de l'Extrait 6 (Myriam) est une bonne élève en classe de terminale, scolarisée dans un lycée classé en ZEP en Seine Saint-Denis ; c'est elle qui a produit le taux moyen le plus élevé de notre test d'écoute.

Le tableau 1 ci-dessous récapitule la liste des locuteurs, leurs degrés moyens de PalAff. L'observation de ces degrés moyens de PalAff de nos stimulus permet de les regrouper (ou de les subdiviser) en trois catégories : extraits ayant un faible degré de palatalisation-affrication (degré I), un degré moyen de palatalisation-affrication (degré II) et un fort degré de PalAff (degré III).

\begin{tabular}{|c|c|c|c|}
\hline \multirow{2}{*}{ Stimulus } & \multirow{2}{*}{ Locuteur } & \multicolumn{2}{|c|}{ PalAff } \\
\hline & & Taux & Degré \\
\hline RFI & Journaliste confirmée & 0,13 & \multirow{2}{*}{ I } \\
\hline Extrait 1 & Virginie, professeure des écoles & 0,19 & \\
\hline Extrait 2 & Valentine, étudiante (Affr moyenne) & 0,55 & \multirow{3}{*}{ II } \\
\hline Extrait 3 & Hugo, jeune journaliste & 0,77 & \\
\hline Extrait 4 & Samia, étudiante algérienne & 0,87 & \\
\hline Extrait 5 & Valentine, étudiante (Affr forte) & 1,30 & III \\
\hline
\end{tabular}




\section{\begin{tabular}{|l|l|l|l|} 
Extrait 6 & Myriam, lycéenne & 1,42 & \\
\hline
\end{tabular}}

Tableau 1. Taux global de PalAff pour chaque extrait.

\subsection{Questions}

Chaque extrait a été écouté deux fois par les auditeurs soumis au test. Pendant et après la deuxième écoute, ils devaient répondre à quelques questions pour évaluer la lecture qu'ils venaient d'entendre, sur une feuille de papier (une feuille par extrait). Nous avons présenté les locuteurs comme des étudiants en école de journalisme, ayant atteint différents niveaux, qui se portaient comme candidats pour lire les informations radiophoniques pour une chaine nationale (généraliste ou culturelle), une chaine communautaire ou pour une radio jeune.

En leur demandant de se mettre à la place d'une personne chargée des recrutements pour la radio, notre première question générale (désormais la « question $A$ ») proposait aux auditeurs d'évaluer la conformité des prestations des candidats avec leurs propres attentes liées à ce métier. Cette question A était formulée ainsi :

En tant que directeur/directrice ou que personne chargée du recrutement des présentateurs de journaux radiophoniques, pensez-vous que cette personne pourrait présenter les informations?

Les auditeurs avaient à choisir entre quatre réponses situées sur une échelle allant du positif vers le négatif (1. tout à fait, 2. plutôt oui, 3. plutôt non, 4. en aucun cas). Les réponses nous ont permis d'obtenir une évaluation globale de chaque lecture en rapport avec ce que serait un présentateur radiophonique proche du prototype de chaque auditeur, et donc d'obtenir un degré d'adéquation aux représentations et attentes des auditeurs au sujet de la prononciation standard. Ainsi, il était possible, par exemple, que les deux journalistes professionnels (la journaliste confirmée et le jeune journaliste) soient globalement mieux évalués que les autres, en dépit de leurs degrés de PalAff différents, ou bien que le jeune journaliste obtienne un score inférieur à celui de la professeure des écoles en raison justement de son taux global de PalAff plus élevé, etc. Il était en outre proposé aux juges d'apporter des précisions sur des points susceptibles d'être modifiés ou améliorés par chaque «candidat » pour que leur production corresponde plus au prototype du journaliste radio.

Les questions suivantes, présentées en bloc, demandaient aux auditeurs de se prononcer de manière plus nuancée sur la possibilité, pour chaque candidat, de présenter des informations dans différents types de radio. Ils devaient tout d'abord répondre à la question : En tant qu'auditeur, trouveriez-vous normal que cette personne présente les infos sur une radio généraliste nationale? (désormais question $\mathrm{RN}$ ). Celle-ci était répétée ensuite en ciblant une radio jeune public (désormais question RJ) et une radio communautaire, visant un public local, ou appartenant à une culture minoritaire (désormais question RC). Les auditeurs avaient à leur disposition la même échelle à quatre degrés, allant de tout à fait jusqu'à en aucun cas, et pouvaient justifier leur réponses en deux lignes.

Enfin, une autre question demandait d'estimer le degré d'aisance de la lecture et la correction de la prononciation, et offrait la possibilité de suggérer des améliorations. Cette question ouverte nous a permis de voir quels étaient les sons dont la prononciation était saillante pour les auditeurs; les réponses obtenues à cette rubrique, assez dispersées, ne seront pas développées dans le présent article.

\subsection{Auditeurs}

80 sujets, dont 50 femmes et 30 hommes, âgés de 15 à 24 ans, ont répondu à ce questionnaire. Parmi eux, 40 habitent en banlieue populaire parisienne et 40 à Grenoble et ses environs.

Les réponses ont été données individuellement mais l'écoute était collective : le protocole a été présenté par l'un ou l'autre des auteurs de manière standardisée devant des groupes de 10 à 20 personnes. Des débats en groupe ont eu lieu immédiatement après la remise des réponses au chercheur présent. 


\section{Résultats}

\subsection{Question A: Perception de l'adéquation des locuteurs au métier de présentateur radio}

La totalité des réponses obtenues à la question $\mathrm{A}$, portant sur l'adéquation globale au métier de présentateur de chaque locuteur (la journaliste de RFI ainsi que les locuteurs des extraits de 1 à 6), est présentée dans le Tableau 2 ci-dessous, en pourcentages.

\begin{tabular}{|c|c|c|c|c|c|c|c|}
\hline Réponse & $\begin{array}{c}\text { RFI } \\
\text { Degré I }\end{array}$ & $\begin{array}{c}\text { E1 } \\
\text { Degré I }\end{array}$ & $\begin{array}{c}\text { E2 } \\
\text { Degré II }\end{array}$ & $\begin{array}{c}\text { E3 } \\
\text { Degré II }\end{array}$ & $\begin{array}{c}\text { E4 } \\
\text { Degré II }\end{array}$ & $\begin{array}{c}\text { E5 } \\
\text { Degré III }\end{array}$ & $\begin{array}{c}\text { E6 } \\
\text { Degré III }\end{array}$ \\
\hline tout à fait & 29 & 60 & 11 & 88 & 3 & 23 & 1 \\
\hline plutôt oui & 65 & 33 & 35 & 10 & 20 & 38 & 26 \\
\hline plutôt non & 6 & 6 & 39 & 2 & 56 & 35 & 47 \\
\hline en aucun cas & 0 & 1 & 15 & 0 & 21 & 4 & 26 \\
\hline Total & 100 & 100 & 100 & 100 & 100 & 100 & 100 \\
\hline tout à fait \& plutôt oui & $\mathbf{9 4}$ & $\mathbf{9 3}$ & $\mathbf{4 6}$ & $\mathbf{9 8}$ & $\mathbf{2 3}$ & $\mathbf{6 1}$ & $\mathbf{2 7}$ \\
\hline plutôt non \& en aucun cas & $\mathbf{6}$ & $\mathbf{7}$ & $\mathbf{5 4}$ & $\mathbf{2}$ & $\mathbf{7 7}$ & $\mathbf{3 9}$ & $\mathbf{7 3}$ \\
\hline
\end{tabular}

Tableau 2. Distribution des réponses obtenues à la question A, en \% arrondis.

La distribution des réponses obtenues est significativement différente du hasard pour chacun des extraits évalués (test khi-2, $\mathrm{p}<0,001$ ). De plus, toujours selon le calcul des khi-2, les distributions des réponses à cette question sont significativement différentes pour chaque extrait si on les compare tous deux à deux (sauf pour la paire Extrait 4 / Extrait 6 pour laquelle les réponses se confondent à toutes les questions, à l'exception de celle concernant les radios jeunes (question RJ).

Nous n'avons trouvé pour la question A aucune corrélation, entre les évaluations obtenues et le taux moyen ou le degré de PalAff: le tableau 3 met en parallèle les extraits et les résultats.

\begin{tabular}{|c|c|c|c|}
\hline \multirow{2}{*}{ Stimulus } & Locuteur & \multicolumn{2}{|c|}{ comparaison } \\
\cline { 2 - 3 } & Hugo, jeune journaliste & Degré de PalAff & Evaluation globale \\
\hline Extrait 3 & Journaliste confirmée & II & \multirow{2}{*}{ positive } \\
\hline RFI & Virginie, professeure des écoles & I & \multirow{2}{*}{ mitigée } \\
\hline Extrait 1 & Valentine, étudiante (Affr forte) & III \\
\hline Extrait 5 & Valentine, étudiante (Affr moyenne) & II & négative \\
\hline Extrait 2 & Myriam, lycéenne & III \\
\hline Extrait 6 & Samia, étudiante algérienne & II \\
\hline Extrait 4 & Tabaut &
\end{tabular}

Tableau 3. Comparaison entre le degré de PalAff et les évaluations. 
Par exemple, malgré son taux de PalAff relativement élevé, c'est l'Extrait 3 (jeune journaliste) qui obtient le plus de réponses positives tout à fait. Ce locuteur rallie donc sans réserve $88 \%$ des auditeurs, ce qui représente un meilleur résultat que les Extraits 1, 2 et RFI, dont les taux moyens de PalAff sont inférieurs.

Si on considère à présent l'Extrait 5, qui présentait le plus fort taux de PalAff de notre échantillon de stimulus, on constate qu'il a reçu un nombre assez faible de réponses très négatives (en aucun cas). En outre, il a reçu plus d'évaluations positives que l'Extrait 2, produit par la même locutrice Valentine) avec un nombre bien inférieur d'occlusives palatalisées ou affriquées. Enfin, l'Extrait 5 a été perçu par les auditeurs comme étant plus proche de ce qui est attendu de la part d'un présentateur radio que l'Extrait 4, lu par une étudiante avec un taux de PalAff plus faible mais avec un léger accent étranger.

\subsection{Perception de l'adéquation des locuteurs au métier de présentateur sur une radio nationale $(R N)$, jeune $(R J)$ ou communautaire (RC)}

Les réponses à la question RN, portant sur l'adéquation des locuteurs pour présenter les informations sur une radio nationale, sont tout à fait cohérentes avec celles obtenues à la réponse A, plus générale et plus vague. Elles sont reportées dans la Figure 2 ci-dessous.

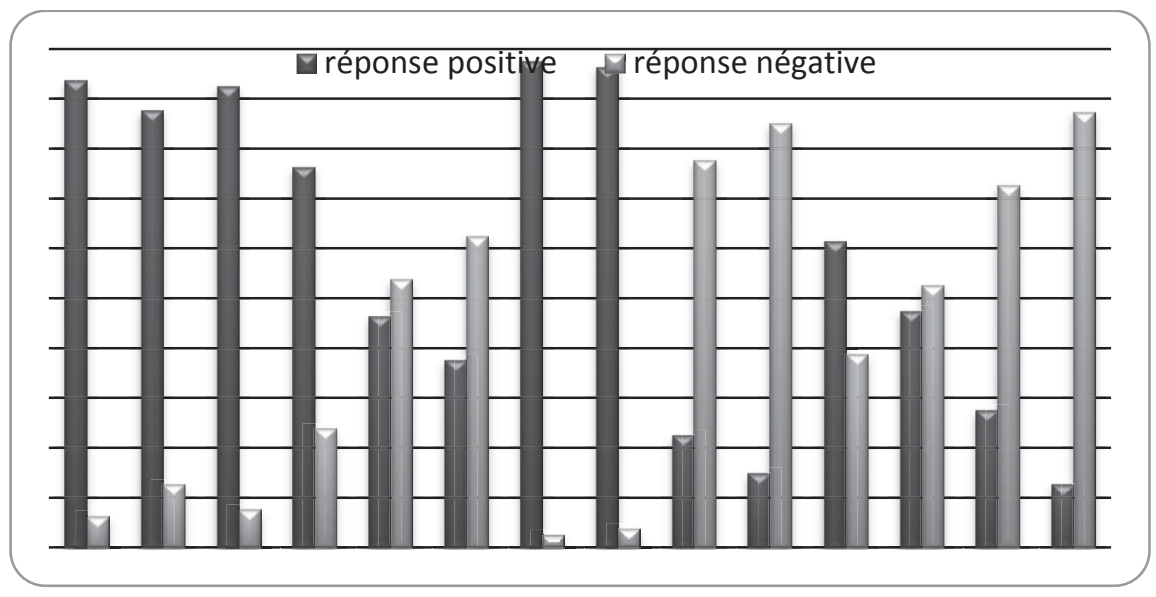

Figure 2: Pourcentages de réponses positives et négatives à la question A comparée à la question RN (radio nationale).

Les évaluations semblent systématiquement légèrement plus sévères que celles données à la réponse $\mathrm{A}$, mais le test khi-2 ne montre pas de différence significative (distributions statistiquement homogènes des réponses à ces deux questions, pour chacun des extraits).

En revanche les réponses obtenues aux questions portant sur la compatibilité des locuteurs avec une radio jeune ou communautaire (RJ et $\mathrm{RC}$ ) ont permis d'affiner un peu ce qui précède. Ces réponses, qui ne sont pas statistiquement différentes entre la question RJ et RC, sont représentées dans la Figure 3 ci-dessous : la hiérarchie du classement n'est pas bouleversée, car les Extraits 1, 3 et 5 obtiennent toujours plus d'évaluations positives que négatives, et inversement pour les Extraits 2, 4 et 6. 


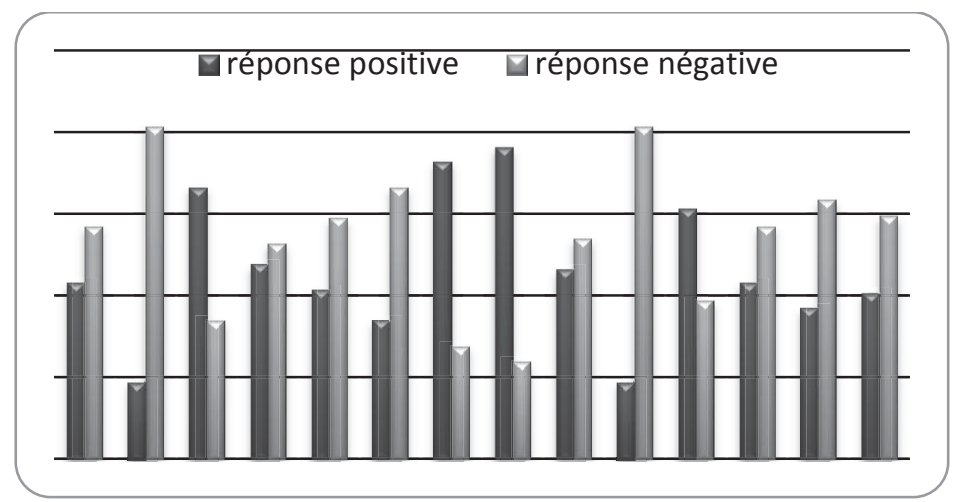

Figure 3: Pourcentages de réponses positives et négatives à la question $R C$ (radio communautaire) comparée à la question $R J$ (radio jeune).

En revanche, nous pouvons remarquer que les évaluations obtenues par l'extrait RFI aux questions RJ et $\mathrm{RC}$ sont inversées par rapport aux deux questions précédentes : il obtient ici plus de réponses négatives que positives, peut-être en raison de son style hautement standardisé et très légitime, trop associé aux radios généralistes et publiques. Il en est de même pour l'Extrait 5, pour lequel les réponses s'inversent également mais uniquement pour la question portant sur la capacité des locuteurs à présenter les informations sur une radio jeune. Les pourcentages d'évaluations négatives diminuent, aux questions RJ et RC, pour les Extraits 4 et 6, tandis qu'ils augmentent pour les Extraits 1, 3 et RFI. Le calcul du khi-2 montre que les réponses obtenues pour les Extraits RFI, 3, 4 et 5 sont différentes pour les questions RN et $\mathrm{RC}$, et également que celles obtenues pour les Extraits 3, 4, 5 et 6 sont différentes pour les questions RN et RJ. L'extrait 2 est évalué à peu près de la même manière (aucune différence significative) quel que soit le type de radio visé.

L'observation de ces résultats montre que les évaluateurs font preuve d'une plus grande tolérance à la variation des prononciations lorsqu'ils écoutent ou s'attendent à écouter une radio jeune ou communautaire, que lorsqu'il s'agit d'une radio généraliste nationale. Ceci aboutit à rapprocher les évaluations des différents extraits entendus, plutôt qu'à les distinguer : contrairement à ce qu'on avait constaté pour les questions $\mathrm{A}$ et $\mathrm{RN}$, la comparaison (khi-2) de tous les extraits pris deux à deux montre que, pour la moitié des paires constituées, les réponses à la question $\mathrm{RC}$ se confondent et ne permettent donc pas de hiérarchiser les extraits.

Aucune différence significative n'a pu être mise en évidence entre les évaluations positives ou négatives produites par les auditeurs de la région parisienne et ceux de la région de Grenoble, ni entre les évaluations produites par les hommes et les femmes, pour aucune des questions posées. Ceci permet de défendre l'hypothèse d'une convergence considérable des réponses à ce type de tâche en France hexagonale, ce qui rejoint les résultats obtenus à un test de perception de grande envergure sur les accents de la francophonie menée par Moreau et alii (2007).

\section{Discussion}

Si l'on s'en tient aux résultats de notre test quantitatif, nous pouvons donc affirmer que dans un contexte formel précis, en l'occurrence la présentation d'informations sur l'antenne d'une radio nationale, les locuteurs professionnels, à savoir, dans notre test, une journaliste confirmée de RFI et un jeune journaliste (Extrait 3), obtiennent globalement des évaluations plus positives, indépendamment de leur degré de PalAff dans la prononciation. Le style de lecture professionnel semble donc avoir plus de poids que le taux de palatalisation/affrication non standard des occlusives. Ils sont suivis immédiatement, dans l'échelle des préférences, par la locutrice professeure des écoles (Extrait 1) dont la prononciation se rapproche le plus du «standard » académique, non seulement en ce qui concerne le taux de PalAff mais aussi sur tous les paramètres. 
Nous pouvons remarquer que l'Extrait 3 a également reçu les appréciations les plus positives pour tous les types de radio suggérés aux auditeurs (jeunes et communautaires, pas seulement généralistes nationales). Il est possible que cette sur-appréciation positive soit favorisée par le fait qu'il s'agissait du seul locuteur homme parmi nos supposés candidats, et/ou par le fait que sa diction corresponde aux exigences professionnelles, sans pour autant être trop académique comme celle de l'Extrait 1 que certains auditeurs ont trouvée un peu précieuse.

Si l'on considère les deux stimulus présentant un taux très élevé de PalAff (extraits 5 et 6), on constate que le premier (5) a reçu des évaluations largement plus positives que le second (6) ; ceci peut être mis en relation avec le fait que l'Extrait 5 était proche des variantes dites standard sur tous les autres paramètres, sauf sur celui de l'affrication qui était forte et systématique, tandis que l'Extrait 6 cumulait d'autres traits considérés comme non standard voire comme allophones (glottalisation de certains /R/, fermeture de quelques nasales $/ \tilde{a} / \ldots)$.

Il est important de souligner que nous n'avons, d'aucune manière que ce soit, attiré l'attention des auditeurs sur le phénomène de palatalisation/affrication, à aucun moment de cette phase de notre expérimentation, ce qui nous permet de penser que les évaluations produites n'ont été biaisées par aucune sorte de focalisation artificielle de l'attention sur les marqueurs étudiés. Bien entendu, les extraits qui ont été soumis aux évaluations, au fond comme n'importe quels échantillons de parole captés dans des conditions naturelles, ne se différencient pas seulement par leurs taux de PalAff, mais également par de nombreuses autres caractéristiques : voix féminine/masculine, timbre jeune/adulte, débit moyen, rapide ou très rapide, présence/absence de petites hésitations, présence/absence d'autres traits non standard comme par exemple des liaisons attendues et non réalisées, des variantes glottalisées de /R/, la présence/absence de l'accent initial caractéristique des lectures radiophoniques professionnelles (cf. Vaissière 1991).

Difficile donc de tirer des conclusions, à partir de notre expérimentation, sur les raisons pour lesquelles l'Extrait 2 (Valentine, taux PalAff moyen) a été globalement moins bien évalué que l'Extrait 5 (Valentine, taux PalAff fort); on pourrait avancer l'hypothèse que le débit de parole plus lent de l'Extrait 2 a été considéré comme moins adéquat pour la lecture de dépêches. En revanche on peut retenir que le poids du taux de PalAff a été plus faible dans l'évaluation globale que celui d'autres marqueurs de la prononciation, qu'il faudra déterminer ultérieurement.

L'absence de toute corrélation entre les taux de PalAff des extraits évalués et les réponses obtenues aux questions $\mathrm{A}$ et $\mathrm{RN}$ notamment, nous incite à formuler la possibilité de généraliser les résultats. Ceux-ci donnent à penser que, dans des circonstances naturelles, lorsque des auditeurs habitant en France hexagonale se trouvent en position d'évaluer la prononciation de journalistes radio en français, la simple présence de phénomènes de PalAff parait ne pas entrainer la perception consciente d'un trait non standard, ou inconvenant pour la radio, ou bien que cette présence serait très peu saillante et jouerait un rôle très secondaire dans l'évaluation par rapport à d'autres marqueurs.

De plus, le fait d'avoir obtenu des réponses significativement différentes, pour la plupart des extraits, entre la question RN d'une part et les questions RJ et RC d'autre part, suggère que les auditeurs sont capables d'adapter finement la notion même de "prononciation standard » en modulant leurs attentes et leurs exigences en fonction du type de radio, et ce tout en restant dans un contexte que l'on s'accorde à considérer comme formel (lectures de dépêches). On peut donc avancer l'hypothèse que la finesse de ces adaptations de critères d'évaluation aux contextes, pourtant à l'œuvre en toutes circonstances, serait complètement gommée par un test de perception qui se contenterait de demander aux sujets s'ils entendent « une prononciation standard » ou bien «quelqu'un qui a un accent particulier », de manière abstraite et décontextualisée ; nous pensons qu'une telle démarche aboutirait à construire des hypothèses sur l'évaluation des variantes de prononciation à partir de données ayant trop peu de rapports avec les pratiques réelles des gens, en situation.

Il reste que ce type d'expérimentation se rapprochant des conditions naturelles souffre d'une véritable limite (cf. Labov 2006) en raison de la difficulté d'isoler un trait unique pour étudier les réactions des sujets qui l'entendent et pour comprendre sa signification sociale. Notre protocole permet de tester si la 
présence du trait agit comme un marqueur particulièrement saillant ou non, mais ne permet pas d'aller plus loin et de tester le poids de chaque unité potentiellement (dé)classante ; ceci impliquerait en effet des manipulations informatiques sur les stimulus et une tâche de induisant une focalisation explicite de l'attention des auditeurs sur un micro-phénomène particulier, ce qui ne correspond pas aux tâches cognitives exécutées spontanément par eux dans des conditions naturelles.

Le constat de ces limites nous incite à plaider pour une forme de mixité méthodologique. Nous pensons qu'il serait nécessaire, pour pouvoir se livrer à des généralisations, de compléter une telle étude par une expérimentation plus ciblée et plus contrôlée, de type matched guise, qui permette d'affiner les interprétations concernant l'existence d'une ou plusieurs significations sociales. Ceci passerait par l'utilisation d'extraits plus courts, s'opposant sur un seul trait, tout en veillant, si possible, à ne pas trop focaliser explicitement l'attention des auditeurs sur le phénomène testé. Néanmoins, nous pensons qu'une telle démarche ciblée sur une seule variable ne pourrait avoir de pertinence qu'en complément, et à la suite, d'un protocole ouvert comme celui que nous avons proposé ici, à compléter d'ailleurs par une démarche qualitative de discussion libre avec les participants. En effet, selon nous, les significations sociales de tel ou tel trait sont très complexes et existent rarement (seulement en cas de stéréotype) en dehors de contextes précis d'interactions entre humains; de plus elles sont susceptibles d'être l'objet de variations interindividuelles importantes (Campbell-Kibler, 2008). Dès lors, il faut d'abord tenter d'observer ou de reconstituer des situations écologiques, d'une durée assez longue, pour pouvoir espérer comprendre de manière globale, dans toute leur complexité, les tendances en matière de perception et catégorisation des locuteurs en fonction de leurs pratiques phonétiques. Ce n'est qu'en se plaçant d'abord dans un tel cadre large, qu'on peut ensuite mener des expérimentations décontextualisées plus ciblées, qu'on ne saurait extrapoler directement sous peine de créer des artefacts, mais qu'on doit pouvoir interpréter au regard des résultats obtenus au préalable, dans des situations naturelles où le phénomène étudié se trouve en co-occurrence avec d'autres phénomènes convergents ou contradictoires.

\section{Conclusions}

Les premiers résultats présentés suggèrent au final que, à ce jour, la simple présence de PalAff dans la prononciation (en principe surveillée) d'une personne en français, en France, ne semble pas suffisamment saillante ou stéréotypique pour qu'elle puisse, à elle seule, susciter des attributions négatives, conscientes ou non, et entrainer des évaluations négatives, au moins de la part des auditeurs âgés de moins de 25 ans. Et ce, même dans des contextes de parole formels et exigeants (en l'occurrence lorsqu'il s'agit d'évaluer la prononciation de locuteurs candidats à la lectures d'informations radiophoniques). Selon nos résultats quantitatifs, le fait de produire des occlusives même assez fortement palatalisées ou affriquées (comme le jeune journaliste de l'extrait 3) ne fait pas partie des critères rédhibitoires pour pouvoir être considéré comme un bon journaliste radiophonique. Ce résultat est convergent avec les données qualitatives dépouillées jusqu'à présent (questions ouvertes et discussions en focus group) car les auditeurs ont rarement exprimé des observations montrant qu'ils ont perçu ces phénomènes. Ceci corrobore également nos observations personnelles préalables sur la présence effective de PalAff, parfois forte, dans la prononciation de journalistes ou autres locuteurs jouissant d'un important prestige social, sans que cela gêne leur carrière.

Dans notre expérimentation, les extraits qui ont suscité les évaluations les plus négatives sont ceux qui présentaient plusieurs autres caractéristiques non standard, perçues globalement comme un accent maghrébin ou jeune et populaire. Il est fort possible que la présence de PalAff contribue à la catégorisation d'une prononciation comme étant non standard et populaire uniquement si elle est suffisamment fréquente et provoque un effet de halo avec d'autres traits, convergents, qu'ils soient linguistiques (prosodie, lexique...) ou non (vêtements, phénotype...).

Ces recherches demandent à être poursuivies et élargies pour le moins dans deux directions : 1) pour établir si la perception de la palatalisation ou affrication est radicalement différente chez des auditeurs plus âgés, toujours en France hexagonale et 2) pour tenter d'éclaircir le poids des différents traits de prononciation dans la catégorisation d'un énoncé comme (in)acceptable dans différents contextes 
considérés comme formels dans la société actuelle. Rien ne nous permet pour le moment d'écarter l'hypothèse d'un possible processus de stéréotypisation à l'œuvre, pour le moins en ce qui concerne l'affrication: si ce processus avance et s'impose, le trait pourrait accéder rapidement à la perception consciente des locuteurs de classe moyenne et supérieure qui pourront le rejeter. En revanche si ce processus n'aboutit pas, le trait pourrait alors se propager rapidement et se diffuser y compris dans la prononciation des locuteurs "légitimes".

\section{Références bibliographiques}

Armstrong, N. \& Pooley, T. Social and Linguistic Change, dans European French, Palgrave Macmillan, 2010.

Campbell-Kibler, K. «I'll be the judge of that: Diversity in social perceptions of (ING) », Language in Society 37, 637-659, 2008.

Castellotti V. \& Robillard D. (de), «Des Français devant la variation : quelques hypothèses », dans Cahiers de l'Institut Linguistique de Louvain 29, Peeters, Louvain-La-Neuve, 223-240, 2003.

Devilla, L. \& Trimaille, C., Variantes palatalisées/affriquées en français hexagonal : quel(s) statut(s) sociolinguistique(s) pour quel destin? dans Iliescu Maria, Siller-Runggaldier H. \& Danler, P. Actes du XXVe Congrès International de Linguistique et de Philologie Romanes, Innsbruck, septembre 2007, Berlin, New York, De Gruyter, vol. 4 : 99-108, 2010.

Fónagy, I., « Le français change de visage? » dans Revue Romane, 24: 225-254, 1989.

Jamin, M., Sociolinguistic Variation in the Paris Suburbs, PhD, University of Kent at Canterbury, 2005

Jamin, M., Trimaille, C. \& Gasquet-Cyrus, M. "De la convergence dans la divergence: le cas des quartiers pluri-ethniques en France”, dans Journal of French Language Studies, 16 : 335-356, 2006. Labov, W., The Social Stratification of English in New York City, $2^{\text {nd }}$ edition, CUP, 2006.

Lehka-Lemarchand, I., Accent de banlieue. Approche phonétique et sociolinguistique de la prosodie des jeunes d'une banlieue rouennaise [thèse Univ. Rouen], 2007.

Lippi-Green R. English with an accent. Language, ideology, and discrimination in the United States, Routledge, 1997.

Morange S. \& Candea M., “Aux frontières de l'écoute. Réflexion sur la construction des variables pertinentes dans la mise en place des tests de perception", dans D. Delomier, (dir), Frontières, $d u$ linguistique au sémiotique, Lambert-Lucas : 79-98, 2010.

Moreau, M.L et alii, 2007, Les accents dans la francophonie. Une enquête internationale. Coll. Français et sociétés, 16, E.M.E et InterCommunications, Belgique.

Ohala J.J. \& Gilbert J.B., "Listeners'Ability to identify Languages by their Prosody", dans Problèmes de prosodie Vol.II, Studia Phonetica 18, Didier, 1979.

Rampton, B. "Styling the Other: Introduction", dans Journal of sociolinguistics, 4: 421-427, 1999.

Trimaille, C. "Who's not palatalizing? Trying to understand the status of palatalized variants in French", 8th Conference of the HDLS, Albuquerque, New Mexico, 6-8 November 2008.

Trimaille C., Consonnes dentales palatalisées/affriquées en français contemporain : indicateurs, marqueurs et/ou variantes en développement ? dans Abecassis, \& Ledegen, G. eds. Les voix des Français : usages et représentations, Actes du colloque AFLS d'Oxford, Septembre 2008, Bern, Peter Lang : 89-100, 2010.

Vaissière J., "Rhythm, accentuation and final lengthening in French", dans Music, Language, Speech and Brain, J. Sundberg, L. Nord, \& R. Carlson. (Eds.) 108-120, 1991.

Vernet, M. \& Trimaille, C. "Contribution à l'analyse de la palatalization en français parlé contemporain", dans Nottingham French Studies. Sociolinguistic Variation and Change in France, Vol. 46, Number $2: 82-99,2007$. 


\subsubsection{Annexe}

Ci-dessous le texte de la dépêche qui a servi de support aux lectures utilisées comme stimulus. Les contextes où une prononciation palatalisée ou affriquée étaient possibles sont soulignés.

A l'occasion du premier anniversaire de la mort de Michael Jackson, les murs de l'institut où il était venu danser quand il avait dix-sept ans ont été recouverts de graffitis et de fresques de peinture à sa gloire, dans la nuit de lundi à mardi. Des fans du chanteur s'étaient en effet donnés rendez-vous sur internet par l'intermédiaire de Facebook. C'est d'ailleurs sur ce réseau social que les autorités ont recueilli les identités des instigateurs présumés de ce rassemblement. La Préfecture de police a promis de punir de telles pratiques de vandalisme. Plusieurs individus ont été interpelés, mais ils se sont défendus d'avoir appelé à graffiter sur les murs de l'institut.

\section{Notes}

\section{Titre de l'article}

Nom, Prénom, \& Nom, Prénom

Affiliation

L'affiliation peut être donnée sur plusieurs lignes

\{premier auteur et deuxième auteur \}@quelquepart.net

Nom, Prénom

Affiliation

Adresse électronique

\section{Titre 1}

Seuls les titres et intertitres utilisent la police Arial ou Helvetica. Tout le reste de l'article est en Times ou Times New Roman.

Tous les paragraphes, à l'exception des titres et intertitres, doivent être justifiés 5 .

\section{Titre 1}

Le corps de texte est en style « Normal» : pas de retrait, Times ou Times New Roman, taille de police 10 pts, justifié, 6 pts avant le paragraphe: l'utilisation de la feuille de style permet d'obtenir automatiquement un espace entre les paragraphes.

\section{Titre 1}

Votre article doit être mis en page de la façon suivante : marge du haut $5 \mathrm{~cm}$, bas, gauche et droite $3 \mathrm{~cm}$. Aucun en-tête, aucun pied de page ne doivent apparaître. Les numéros de page seront ajoutés automatiquement au moment de la publication des actes : merci de bien vouloir vérifier que vous n'en avez pas inséré.

\subsection{Titre 2}

Les citations courtes sont insérées entre guillemets dans le corps de texte : les citations longues peuvent être distinguées par l'emploi du style « Citation»: 


\begin{abstract}
Citation longue Citation longue Citation longue Citation longue Citation longue Citation longue Citation longue Citation longue Citation longue Citation longue Citation longue Citation longue Citation longue Citation longue Citation longue Citation longue Citation longue Citation longue Citation longue Citation longue Citation longue Citation longue Citation longue Citation longue Citation longue Citation longue
\end{abstract}

\title{
8.1.1 Titre 3
}

Il est préférable de ne pas créer plus de trois niveaux de titres / sections.

\section{Tableaux et graphiques}

Les titres des tableaux doivent être en style "Normal" : Times ou Times New Roman, taille de police 10, justifié.

Les titres des tableaux et graphiques doivent constituer deux listes numérotées indépendamment (par exemple : Tableau 1, Tableau 2, Tableau 3, Graphique 1).

Vous pouvez également créer des listes avec puces en utilisant le style « Hiérarchisation, 9pt »

- Exemple

- Exemple Exemple

- Exemple Exemple Exemple

\section{Envoi de votre article}

Votre article doit être déposé en ligne, sur la plateforme prévue à cet effet.

\subsection{Format du document}

Votre article doit être envoyé au format .doc

\section{Références bibliographiques (style "Titre 1 - Centered" ; mêmes caractéristiques que le style prédéfini par Word "Titre 1", mais centré et sans numérotation : Arial ou Helvetica, taille de police 12, gras)}

Pour la bibliographie, Times ou Times New Roman, taille de police 9, justifié. La première ligne de chaque référence (donc la première ligne de chaque paragraphe) doit être affectée d'un retrait négatif de $0,5 \mathrm{~cm}$. Vous trouverez cidessous le format des références pour les articles et les ouvrages.

Nom de l'auteur, Initiale du prénom. (année de publication). Titre de l'article. Nom de la revue, Numéro de la revue, numéros des pages.

Nom de l'auteur, Initiale du prénom. (Année de publication). Titre de l'ouvrage. Ville : Editeur.

Pour tout renseignement complémentaire : cmlf08@ivry.cnrs.fr

\footnotetext{
${ }^{1}$ Nous avons bien conscience du caractère éminemment problématique de cette catégorie qui a toutefois l'avantage de permettre d'éviter de longues périphrases sociologiquement plus exactes.

${ }^{2}$ Ce néologisme référant à une attente de productions non marquées, ni géographiquement, ni socialement.
} 
${ }^{3}$ Nous remercions Jean-Pierre Lai, du Gipsa-Lab, (Université Stendhal Grenoble 3 et Grenoble INP).

${ }^{4}$ L'ordre de la passation du test d'écoute n'était ni croissant, ni décroissant, pour ne pas induire des attentes précises de la part des auditeurs.

${ }^{5}$ Votre document ne doit comporter ni en-tête ni pied de page. Les notes sont donc des notes de fin de document, non des notes de bas de page. 